\title{
ESTIMANDO E IDENTIFICANDO NA CLASSE DE MODELOS LINEARES HIERÁRQUICOS NORMAIS
}

\author{
Eliane Amiune Camargo ${ }^{1}$ \\ Dani Gamerman²
}

\section{Resumo}

Este traballıo tem como oljjetivo discntir problemas de estimação, identificação e previsão que ocorrem en algms moclelos lineares hierárquicos normais. Inicialmente esta cliscussão é feita para o moclelo polinomial cle $1 \underline{a}$ orclem tanto em sna forma estática quanto dinânica. Baseado nesta análise, conclnsõos foran tiraclas e extensões foran feitas através cle nnistnra cle clistrilııições (Ganermm \& S'nithlı, 1996) cle moclo a tornar o moclelo iclentificável possibilitando assinn mua mellıor estimação clos parâmetros. Levanclo-se en contcl que este moclelo pocle ser visto como um elemento geraclor cla classe geral cle Moclelo Linear Normal Dinânico (DLMs), generalizações foram feitas para modelos polinomiais cle orclem $k$ e moclelos cle regressão.

\section{Abstract}

The oljjective of this paper is to examine problenss of estination, identification and prevision that occur in some normal hierarchical linear moclels. Firstly this discnssion was for the first-order polynomial moclel both in its static and clynamic shape. Based on that analysis some conclusions were obtained and extensions were macle from the mixtme of clistrilsutions (Ganerman \& S Smith, 1996) in order to maintain the moclel identifiable with the possibility of a best estimation of the paraneters. Consiclering this moclel as a generator element of the general class of Dynamic Normal Linear Moclel (DLMs), generalizations were macle for polynomial moclels of orcler $k$ and regression models.

1 Instituto de Matemática, Universidade Federal do Rio de Janeiro, Cx. Postal 6Sā30, 21944, Rio de .Janeiro, R.J, Brasil. E-mail: elianel o.pinı.nfr.j.ur

2 Instituto de Matemática, Universidade Federal do Rio de .Janeiro, Cx. Postal 6S5̃30. 21044, Rio cle .Janeiro: R.J, Brasil. E-mail: dani dunenfri.jur

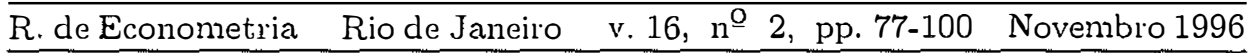


Estimando e identificando na classe de modelos lineares hierárquicos normais

Palavras.Chave: Estimação, identificação, previsão, mistura de distribuiçōes.

Código JEL: C32, C11

\section{Introdução.}

Neste artigo analisamos problemas de identificação, previsão e estimação que ocorrem em alguns modelos lineares hierárquicos normais. Inicialmente detectamos estes problemas no modelo polinomial de $1 \underline{\text { a }}$ ordem e mostramos que modelos atuariais e econométricos analisados respectivamente por Klugman (1992), Marshall (1991) e Harvey (1989) quando colocados na forma hierárquica podem ser vistos como variações deste modelo.

Semelhanças entre modelos hierárquicos e modelos de componentes de erro são conhecidas na literatura, e podemos ressaltar que este último tem sido usado em econometria desde os trabalhos de Balestra e Nerlove (1966) e na literatura estatística é conhecido como modelo de Análise de Variância. Tanto o modelo de Fatores Comuns (Harvey, 1989) quanto o modelo de Componentes de Erro (Marshall, 1991) apresentam problemas de identificabilidade e estes foram contornados impondo restrições ao modelo como será visto posteriormente.

A contribuição deste artigo está na utilização de mistura de distribuições (Gamerman \& Smith, 1996) para tornar o modelo identificável, possibilitando assim uma melhor estimação dos parâmetros.

$\mathrm{Na}$ próxima Seção faremos uma rápida introdução a modelos lineares hierárquicos normais e apresentaremos o modelo polinomial de $1^{\underline{L}}$ ordem, modelo $M_{1}$, que embora seja o mais simples modelo de regressão, pode ser visto como um elemento gerador da classe de Modelo Linear Normal Dinâmico (DLMs). Na Seção 3, apresentamos com detalhe os modelos estudados por Klugman (1992), Marshall (1991) e Harvey (1989) que, como foi dito acima, são variações do modelo $M_{1}$. Na Seção 4, analisamos o modelo $M_{1}$ tanto em sua forma estática quanto dinâmica, ressaltando o problema de estimação 
da variância. Finalmente na Seção 5 , generalizamos o modelo $M_{1}$ usando mistura de distribuições e na Seção 6 apresentamos modelos alternativos que são extensões de modelos polinomiais de ordem $\mathrm{k} e$ modelos de regressão.

\section{Modelo Hierárquico.}

A macroeconomia atual utiliza cada vez mais métodos de séries temporais (Issler, 1992) e a abordagem Bayesiana pode ser aplicada eficientemente na análise de tais séries (Zellnes: 1971). Modelo linear hierárquico é uma extensão de modelo linear normal e permite modelar tendências estocásticas que é o caso de muitas séries temporais econômicas. Um fenômeno econômico envolve muitas variáveis e é importante que ele seja modelado de modo a incorporar as relações existentes entre estas variáveis, o que pode ser feito através de uma modelagem hierárquica. O modelo linear hierárquico normal originalmente introduzido por Lindley \& Smith (1972) tem a forma:

$$
\begin{gathered}
Y \mid \Theta, \overline{V_{1}} \sim N\left(F_{1} \Theta, V_{1}\right) \\
\Theta \mid \mu, V_{2} \sim N\left(F_{2} \mu, V_{2}\right) \\
\Pi\left(\mu, V_{1}: V_{2}\right)
\end{gathered}
$$

onde

$Y_{n \times 1}$ é um vetor contendo os dados,

$\Theta_{k \times 1}$ é o vetor de parâmetros de interesse,

$\left(F_{1}\right)_{n \times k},\left(F_{2}\right)_{k \times p}$ matrizes conhecidas,

$(\mu)_{p \times 1}$ é vetor de hiperparâmetros,

$\left(V_{1}\right)_{n \times n,},\left(V_{2}\right)_{k \times k}$ matrizes de covariância positiva definida desconhecidas,

$\Pi$ especifica uma priori para $\mu, V_{1}$ e $V_{2}$. 
Estimando e identificando na classe de modelos lineares hierárquicos normais

Permitindo o processo acima evoluir ao longo do tempo, obtemos um Modelo Normal Linear Dinâmico Hierárquico.

$$
\begin{aligned}
Y_{t} \mid \Theta_{t,}, V_{1 t} & \sim N\left(F_{1 t} \Theta_{t}, V_{1 t}\right) \\
\Theta_{t} \mid \mu_{t}, V_{2 t} & \sim N\left(F_{2 t} \mu_{t,}, V_{2 t}\right), \quad l=1,2, \cdots \\
\mu_{t,} \mid \mu_{t-1}, W_{t} & \sim N\left(G_{t, \mu_{t-1}}, W_{t}\right)
\end{aligned}
$$

onde $V_{1 t}, V_{2 t}$. podem ser ou não conhecidas.

Introduzimos assim, uma incerteza adicional, uma vez que ao contrário dos modelos estáticos, o futuro não será como o passado.

Para que decisões sejam tomadas em tais modelos, é necessário que a estruturação do modelo hierárquico modele os níveis de hierarquia sem perder de vista a relação entre eles o que pode acarretar um problema de identificação, como veremos a seguir.

Outro problema que surge na construção de modelos é o da previsão. Para fazermos previsão sobre alguma quantidade (ou coleção de quantidades) de interesse, sob o ponto de vista Bayesiano, é necessário o conhecimento de informações previstas em termos da distribuição que representam e sumarizam collhecimentos incertos.

Inferência Bayesiana é baseada na premissa fundamental que todas as incertezas são representadas e medidas por probabilidades. Todas as probabilidades são subjetivas.

O modelo linear normal hierárquico dinâmico de $1 \underline{a}$ ordem, modelo $M_{1}$, apesar de ser o mais simples modelo de regressão, ilustra conceitos básicos e características importantes de classe geral de Modelo Linear Normal Dinâmico (DLMs). Sendo assim, nos proporciona uma base para futuras generalizações.

Iniciaremos nossa discussão com o modelo polinomial de $1 \stackrel{a}{a}$ ordem, modelo $M_{1}$, dado por

$$
\begin{aligned}
y_{t i} & =\mu_{t, i}+\varepsilon_{t, i} \\
\mu_{t, i} & =\mu_{t}+\eta_{t, i,}, \quad t=1,2, \cdots \quad i=1, \cdots, n \\
\mu_{t} & =\mu_{t-1}+\omega_{t .}
\end{aligned}
$$


Eliane A. Camargo \& Dani Gamerman

onde

$\underline{y_{t}}=\left(y_{t, 1}, y_{t .2}, \cdots, y_{t . n .}\right)^{T}$ é o vetor de observações no tempo $t$,

$\underline{\mu_{t .}}=\left(\mu_{t .1}, \mu_{t, 2}, \cdots, \mu_{t . n .}\right)^{T}$ é o vetor de médias das observações no tempo $t$,

$\varepsilon_{t i}, \eta_{t, i}$ e $w_{t}$ são serialmente e mutuamente não correlacionados.

Das equações (2.1) temos que as observações $y_{t i}^{\prime} s$ variam em torno de níveis $\mu_{t i}^{\prime} s$ que são permutáveis com média comum $\mu_{t}$, sujeita a um passeio aleatório ao longo do tempo $t$.

A característica básica deste modelo, é que projetando $k$ passos para o futuro, o valor esperado da série condicional ao conhecimento no tempo $t$, é simplesmente

$$
E\left(\underline{y}_{t+k} \mid \underline{\mu}_{t}\right)=E\left(\underline{\mu}_{t+k} \mid \underline{\mu}_{t}\right)=\underline{\mu}_{t}
$$

Consequentemente a função de previsão é

$$
f_{t}(k)=E\left(\underline{y}_{t+k} \mid D_{t}\right)=E\left(\underline{\mu}_{t} \mid D_{t}\right)=h_{t} 1_{n,}, k>0
$$

onde $1_{n}$ é o vetor n-dimensional de $1^{\prime} s, D_{t}$, é a informação disponível no tempo t e $h_{t}=E\left(\mu_{t} \mid D_{t}\right)$.

Portanto, o nível futuro da série é constante e assim tal modelo só é útil para aplicação de curto-prazo.

Combinando as duas primeiras equações do modelo $M_{1}$, podemos reescrever a equação das observações na forma:

$$
\underline{y}_{t}=1_{n} \mu_{t}+\underline{\eta}_{t}+\underline{\varepsilon}_{t}
$$

portanto só conseguimos estimar $\underline{\eta}_{t}+\underline{\varepsilon}_{t}$ das observações evidenciando assim o problema de estimação das variâncias que será analisado posteriormente. 
Estimando e identificando na classe de modelos lineares hierárquicos normais

\section{Variações do Modelo $M_{1}$.}

Apresentaremos agora alguns modelos que podem ser vistos como variações do modelo $M_{1}$.

Klugman (1992) analisou o modelo

$$
\begin{aligned}
& y_{t, i}=\mu_{t, i}+\varepsilon_{t, i}, \quad \varepsilon_{t, i} \sim N\left(0, \sigma^{2} / P_{t, i}\right) \\
& \mu_{t, i}=\rho \mu_{t,-1, i}+(1-\rho) \eta_{i}+v_{t, i,}, \quad v_{t, i} \sim N\left(0, \gamma^{2}\right) .
\end{aligned}
$$

Neste modelo, as componentes de $\mu_{t}$ são julgadas não permutáveis com média que é uma combinação de evoluções individuais e efeitos individuais estáticos. Uma característica interessante deste modelo é a variância incondicional (com respeito a $\mu_{t i}$ ) de uma observação

$$
\begin{aligned}
V\left[y_{t i} \mid\right. & \left.\eta_{i}, \rho, \sigma^{2}, \gamma^{2}\right]=\operatorname{Var}\left[E\left(y_{t i} \mid \mu_{t i,}, \eta_{i}, \rho, \sigma^{2}, \gamma^{2}\right)\right] \\
+ & E\left[\operatorname{Var}\left(y_{t i .} \mid \mu_{t, i}, \eta_{i, \rho}, \sigma^{2}, \gamma^{2}\right)\right] \\
& =\operatorname{Var}\left[\mu_{t, i} \mid \eta_{i}, \rho, \sigma^{2}, \gamma^{2}\right]+E\left[\sigma^{2} / P_{t, i} \mid \eta_{i}, \sigma^{2}, \gamma^{2}, \rho\right] \\
& =\gamma^{2}+\sigma^{2} / P_{t i} .
\end{aligned}
$$

Temos dois casos de especial interesse nesse modelo:

1ㅇ) Se $\rho=0$, obtemos

$$
\left\{\begin{array}{cc}
y_{t, i}=\mu_{t, i}+\varepsilon_{t i,} & , \quad \operatorname{Var}\left(\varepsilon_{t, i}\right)=\sigma^{2} / P_{t, i} \\
\mu_{t, i}=\eta_{i}+v_{t i} & , \quad \operatorname{Var}\left(v_{t, i}\right)=\gamma^{2}
\end{array}\right.
$$

Neste caso, cada parâmetro no tempo $t$ pode ser considerado como uma observação aleatória da distribuição com média nãopermutável.

Fazendo $i=1, \cdots, k, t=1, \cdots, n_{i}$ e $\sum n_{i}=N$ (total de observações) obtemos o modelo de Análise de Variância, onde no 1ㅇ 
nível temos $n_{i}$ observações para cada um dos $k$ grupos. Esse modelo corresponde ao analisado por Buhlmann e Straub (1972) e Meyers (1989), importante no contexto atuarial pois permite diferentes variâncias para diferentes grupos, assegura o mesmo valor para cada grupo e relaciona que o risco de perda $y_{t, i}$ é inversamente proporcional ao prêmio $P_{t, i}$.

2 으) Se $\rho=1$, obtemos:

$$
\left\{\begin{array}{cc}
y_{t, i}=\mu_{t, i}+\varepsilon_{t, i} & , \quad \operatorname{Var}\left(\varepsilon_{t, i}\right)=\sigma^{2} / P_{t, i} \\
\mu_{t, i}=\mu_{t,-1, i}+v_{t, i} & , \quad \operatorname{Var}\left(v_{i, t}\right)=\gamma^{2}
\end{array}\right.
$$

Aqui, cada parâmetro no tempo $t$ é o parâmetro no tempo anterior mais um ajuste aleatório. As séries são nâo correlacionadas.

Marshall (1991) considerou uma extensâo dinâmica para o modelo clássico de componente de erro. A principal motivação do modelo analisado por ele está no fato de que muitas séries temporais econômicas são estocásticas apesar da tendência determinística comum e, assim não podem ser representadas por estruturas estacionárias (Nelson e Plosser, 1982).

O modelo clássico de componente de erro é descrito por

$$
\begin{gathered}
y_{t i,}=\mu+\lambda_{i}+v_{t}+\omega_{t, i} \\
i=1, \cdots, n \\
t=1, \cdots, T
\end{gathered}
$$

onde

$y_{t, i}$ é a observação da unidade $i$ no tempo $t$,

$\mu$ é a média global,

$\lambda_{i}$ é o efeito de unidade específica,

$v_{t}$ é o efeito de tempo específico, 
Estimando e identificando na classe de modelos lineares hierárquicos normais

$\omega_{t, i}$ é o efeito específico de unidade-tempo,

$E\left(\lambda_{i}\right)=E\left(v_{t}\right)=E\left(\omega_{t, i}\right)=0$,

$\lambda_{i}, v_{t}, \omega_{t, i}$ são serialmente e mutuamente não-correlacionados.

Permitindo $v_{l}$ e $\omega_{t, i}$ evoluirem no tempo como um passeio aleatório, o modelo (3.1) pode ser reescrito na forma

$$
\begin{aligned}
& y_{t, i}=\mu_{t, i}+\varepsilon_{t}+\varepsilon_{t i}^{*} \\
& \mu_{t, i}=\mu_{t,-1, i}+\eta_{t}+\eta_{t, i}^{*}
\end{aligned}
$$

onde os distúrbios $\varepsilon_{t,}, \varepsilon_{t, i}^{*}, \eta_{t,}, \eta_{t, i}^{*}$ são normalmente distribuídos e mutuamente não correlacionados com méclia zero e respectivas variâncias

$$
\begin{aligned}
& \operatorname{Var}\left(\varepsilon_{t}\right)=\sigma_{\varepsilon}^{2} ; \quad \operatorname{Var}\left(\varepsilon_{t i}^{*}\right)=\sigma_{\varepsilon^{*}}^{2} \quad \mathrm{e} \\
& \operatorname{Var}\left(\eta_{t}\right)=\sigma_{\eta}^{2} ; \quad \operatorname{Var}\left(\eta_{t, i}^{*}\right)=\sigma_{\eta^{*}}^{2} .
\end{aligned}
$$

Como um modelo séries temporais multivariado n-dimensional o modelo (3.2) pode ser reescrito na forma

$$
\begin{aligned}
& \underline{y}_{t}=\underline{\mu}_{t}+1_{n} \varepsilon_{t}+\underline{\varepsilon}_{t}^{*} \\
& \underline{\mu}_{t}=\underline{\mu}_{t-1}+1_{n} \eta_{t}+\underline{\eta}_{t}^{*}
\end{aligned}
$$

onde

$1_{n}$ é um vetor $n \times 1$ de 1 's,

$\varepsilon_{t}$ e $\eta_{t}$ são os efeitos comuns do choque aleatório,

$\varepsilon_{t:}^{*}=\left(\varepsilon_{t, 1}^{*}, \cdots, \varepsilon_{t, n}^{*}\right)$ e $\eta_{t:}^{*}=\left(\eta_{t: 1}^{*}, \cdots, \eta_{t, n}^{*}\right)$ são os efeitos específicos do choque aleatório.

Um caso particular de especial interesse é obtido fazendo $\sigma_{\eta}^{2}>0$ e $\sigma_{\eta^{*}}^{2}=0$ em (3.2), a saber

$$
\begin{aligned}
& y_{t, i}=\mu_{t,}+\lambda_{i}+\varepsilon_{t}+\varepsilon_{t, i}^{*} \\
& \mu_{t}=\mu_{t-1}+\eta_{t}
\end{aligned}
$$


onde $\mu_{t}$, é a média comum e, para que o modelo seja identificável precisamos ter $\sum \lambda_{i}=0$. O modelo (3.4) corresponde a uma variação do caso $\rho=1$ do modelo analisado em Klugman (1992). Neste modelo temos que as $n$ séries a menos de um efeito invariante no tempo tem a mesma média dependente no tempo, ou seja, as n-séries são cointegradas no sentido de Engle e Granger (1987). A vantagem da formulação (3.2) e (3.4) é o uso da estrutura de correlação serial do processo para obter estimadores mais eficientes da média no tempo $t$; usando não somente a informação atual, mas também a informação em períodos anteriores.

Harvey (1989) além de estudar as propriedades dos modelos (3.2) e (3.4), analisou o modelo de fatores comuns dado pelas equações abaixo

$$
\begin{aligned}
& y_{t}=\Theta \mu_{t}+\mu_{0}+\varepsilon_{t,}, \quad V\left(\varepsilon_{t}\right)=\Sigma_{\varepsilon} \\
& \mu_{t}=\mu_{t-1}+\beta+\eta_{t,}, \quad V\left(\eta_{t,}\right)=\Sigma_{\eta_{l}}
\end{aligned}
$$

onde

$\Theta=\left[\theta_{i, j}\right]$ é a matriz de peso $n \times k, k \leq n, 1 \leq i \leq n, 1 \leq j \leq k$, $\mu_{t}$ é o vetor de tendência comum $k \times 1$,

$\Sigma_{\varepsilon}$ é a matriz de covariância $n \times n$ positiva definida, $\Sigma_{\eta}$ é a matriz de covariância $k \times k$ positiva definida,

$\mu_{0}$ é o vetor com os $k$ primeiros elementos nulos e $(n-k)$ elementos irrestritos.

O modelo (3.5) apresenta vantagens como:

i) ser mais parcimonioso que o modelo sem tendência comum;

ii) preservar certos níveis de relação entre as variáveis quando previsões são feitas;

iii) ter uma interpretação interessante para a tendência comum depois de uma rotação apropriada. 
Estimando e identificando na classe de modelos lineares hierárquicos normais

Entretanto, tal como ele se apresenta, não é identificável. Este problema de identificabilidade é contornado impondo restrições à matriz de pesos comuns $\Theta$ e a matriz de covariãncia $\Sigma_{\eta}$. As imposições feitas por Harvey foram:

i) $\Sigma_{\eta}$ é uma matriz diagonal;

ii) $\theta_{i, j}=0, j>i$;

iii) $\theta_{i i}=1, i=1, \cdots, k$.

Estas restrições impostas à matriz $\Theta$, permitem que as tendências comuns sejam não correlacionadas umas com as outras, gerando desta forma $(n-k)$ combinações lineares estacionárias, o que segundo Engle e Granger define um modelo cointegrado.

Considerando em particular $\Theta=I_{n}$ e $\mu_{0}=0_{n}$ em (3.5) obtemos o modelo $M_{1}$. Na seção seguinte analisamos o modelo $M_{1}$, evidenciando mais uma vez o problema de estimação de variância existente e propomos uma solução alternativa que basicamente equivale a uma reparametrização dos modelos analisados por Marshall e Harvey.

\section{Análise do Modelo $M_{1}$.}

Retornaremos agora ao modelo $M_{1}$, para analisá-lo tanto em sua forma estática quanto dinâmica e ver que possíveis generalizações do mesmo permitem uma melhor estimação dos parâmetros.

Consideremos a versão estática do modelo $M_{1}$ :

$$
\begin{array}{llll}
y_{i} & =\mu_{i}+\varepsilon_{i} & , & \varepsilon_{i} \sim N\left(0, V_{1}\right) \\
\mu_{i} & =\mu+v_{i} & , & v_{i} \sim N\left(0, V_{2}\right) \\
\mu & \sim N(a, R) &
\end{array}
$$

onde $\varepsilon_{i}, v_{i}$ são serialmente e mutuamente não correlacionados e $V_{1} \mathrm{e}$ $V_{2}$ são conhecidas. 
Eliane A. Camargo \& Dani Gamerman

Para o modelo acima temos que a verossimilhança pode ser escrita na forma

$$
\begin{aligned}
p\left(y \mid \underline{\mu}, \mu, V_{1}, V_{2}\right) & =\prod_{i=1}^{n} p\left(y_{i} \mid \mu_{i}, V_{1}\right) p\left(\mu_{i} \mid \mu, V_{2}\right) \\
\alpha & \prod_{i=1}^{n} \exp \left\{-\frac{1}{2} \frac{\left(y_{i}-\mu_{i}\right)^{2}}{V_{1}}\right\} \exp \left\{-\frac{1}{2} \frac{\left(\mu_{i}-\mu\right)^{2}}{V_{2}}\right\}
\end{aligned}
$$

onde $\underline{\mu}=\left(\mu_{1}, \mu_{2}, \cdots, \mu_{n}\right)$.

Derivando a $\log$ verossimilhança $L\left(\underline{\mu}, \mu, V_{1}, V_{2}\right)=\log [p(u \mid$ $\left.\left.\underline{\mu}, \mu, V_{1}, V_{2}\right)\right]$ em relação a $\mu_{i}$ e $\mu$ e denotando por $\hat{\mu}_{i}$ e por $\hat{\mu}$ os respectivos estimadores de máxima verossimilhança temos

$$
L\left(\underline{\mu}, \mu, V_{1}, V_{2}\right)=c-\frac{1}{2} \sum_{i=1}^{n} \frac{\left(y_{i}-\mu_{i}\right)^{2}}{V_{1}}-\frac{1}{2} \sum_{i=1}^{n} \frac{\left(\mu_{i}-\mu\right)^{2}}{V_{2}}
$$

assim

$$
\begin{aligned}
& \frac{\partial L}{\partial \mu_{i}}\left(\underline{\mu}, \mu, V_{1}, V_{2}\right)=\frac{\left(y_{i}-\mu_{i}\right)}{V_{1}}-\frac{\left(\mu_{i}-\mu\right)}{V_{2}} \\
& \frac{\partial L}{\partial \mu}\left(\underline{\mu}, \mu, V_{1}, V_{2}\right)=\sum_{i=1}^{n} \frac{\left(\mu_{i}-\mu\right)}{V_{2}}
\end{aligned}
$$

e portanto

$$
\frac{\partial L}{\partial \mu}\left(\underline{\mu}, \mu, V_{1}, V_{2}\right)=0 \Leftrightarrow \sum_{i=1}^{n} \mu_{i}=n \mu \Leftrightarrow \hat{\mu}=\bar{\mu} .
$$

Substituindo (4.3) em (4.2) obtemos

$$
\frac{\partial L}{\partial \mu_{i}}\left(\underline{\mu}, \hat{\mu}, V_{1}, V_{2}\right)=0 \Leftrightarrow \frac{y_{i}-\mu_{i}}{V_{1}}=\frac{\mu_{i}-\bar{\mu}}{V_{2}}
$$

Revista de Econometria 16 (2) Novembro 1996 
Estimando e identificando na classe de modelos lineares hierárquicos normais

o que nos dá

$$
\begin{array}{rlc}
\hat{\mu} & = & \bar{\mu} \\
\hat{\mu}_{i} & = & \frac{V_{2} y_{i}+V_{1} \mu}{V_{1}+V_{2}} .
\end{array}
$$

Por outro lado, temos de $\frac{\partial L}{\partial \mu_{i}}=0$ que

$$
\sum_{i=1}^{n} \frac{\left(y_{i}-\mu_{i}\right)}{V_{1}}=\sum_{i=1}^{n} \frac{\left(\mu_{i}-\vec{\mu}\right)}{V_{2}} .
$$

Como $\sum_{i=1}^{n} \frac{\left(\mu_{i}-\bar{\mu}\right)}{V_{2}}=0$, temos $\bar{y}=\bar{\mu}$ e consequentemente

$$
\begin{aligned}
\hat{\mu}_{i} & =\frac{V_{2} y_{i}+V_{1} \bar{y}}{V_{1}+V_{2}} \\
& =(1-p) y_{i}+p \bar{y}
\end{aligned}
$$

onde $p=V_{1} /\left(V_{1}+V_{2}\right)$.

Consequentemente,

$$
L\left(\underline{\hat{\mu}}, \hat{\mu}, V_{1}, V_{2}\right)=c-\frac{1}{2} \sum_{i=1}^{n} \frac{\left(y_{i}-\bar{y}\right)^{2}}{V_{1}+V_{2}} .
$$

Segue portanto de (4.7) que $L\left(\hat{\mu}, \hat{\mu}, V_{1}, V_{2}\right)$ depende de $V_{1}$ e $V_{2}$ através de sua soma, sendo assim só conseguimos estimar $V_{1}+V_{2}$ das observações.

Vale observar que

$$
E\left(\mu_{i} \mid \underline{y}\right)=E\left(E\left(\mu_{i} \mid \mu, \underline{y}\right)\right)
$$

onde

$$
\mu_{i} \mid \mu, \underline{y} \sim N\left(\frac{V_{2} y_{i}+V_{1} \mu}{V_{1}+V_{2}}, V_{1}^{-1}+V_{2}^{-1}\right) .
$$


Eliane A. Camargo \& Dani Gamerman

Assim

$$
E\left(\mu_{i} \mid \mu, \underline{y}\right)=\frac{V_{2} y_{i}+V_{1} \mu}{V_{1}+V_{2}}
$$

e consequentemente

$$
\begin{aligned}
E\left(\mu_{i} \mid \underline{y}\right) & =E\left(\frac{V_{2} y_{i}+V_{1} \mu}{V_{1}+V_{2}} \mid \underline{y}\right) \\
& =\frac{V_{2} y_{i}}{V_{1}+V_{2}}+\frac{V_{1}}{V_{1}+V_{2}} E(\mu \mid \underline{y}) \\
& =(1-p) y_{i}+p E(\mu \mid \underline{y}) .
\end{aligned}
$$

Por outro lado,

$$
\mu \mid \underline{y} \sim N\left(\frac{R \bar{y}+\left(V_{1}+V_{2}\right) a}{R+V_{1}+V_{2}},\left(V_{1}+V_{2}\right)^{-1}+R^{-1}\right)
$$

e assim

$$
\begin{aligned}
E(\mu \mid \underline{y}) & =\frac{R \bar{y}+\left(V_{1}+V_{2}\right) a}{R+V_{1}+V_{2}} \\
& =q a+(1-q) \bar{y}
\end{aligned}
$$

onde $q=V_{1}+V_{2} /\left(R+V_{1}+V_{2}\right)$, e finalmente

$$
E\left(\mu_{i} \mid \underline{y}\right)=(1-p) y_{i}+p[q a+(1-q) \bar{y}] .
$$

Observemos também que $E\left(\mu_{i} \mid \underline{y}\right) \rightarrow \hat{\mu_{i}}$ quando $R \rightarrow \infty$, coincidindo com o que seria obtido considerando uma priori difusa para $\mu$ no modelo (4.1).

Uma solução alternativa para o problema de estimação que ocorre no modelo estático (4.1), é considerar uma mistura para as médias, obtendo o modelo

$$
\begin{aligned}
y_{i} & =\mu_{i}+\varepsilon_{i}, \quad \varepsilon_{i} \sim N\left(0, V_{1}\right) \\
\mu_{i} & \sim(1-p) N\left(a_{i}, R_{i}\right)+p \mu \\
\mu & \sim N(a, R) .
\end{aligned}
$$


Estimando e identificando na classe de modelos lineares hierárquicos normais

Usando variável indicadora $k$, podemos reescrever (4.9) na forma

$$
\begin{aligned}
y_{i} & =\mu_{i}+\varepsilon_{i} \\
\mu_{i} & =(1-k)\left(a_{i}+v_{i}\right)+k \mu \\
\mu & \sim N(a, R) \\
k & \sim \operatorname{Ber} n(p) \\
v_{i} & \sim N\left(o, R_{i}\right) .
\end{aligned}
$$

Desta forma o modelo (4.9) oscila entre

$$
\begin{aligned}
y_{i} & =\mu_{i}+\varepsilon_{i} \\
\mu_{i} & =\mu \\
\mu & \sim N(a, R)
\end{aligned}
$$

com probabilidade $p$ e

$$
\begin{aligned}
& y_{i}=\mu_{i}+\varepsilon_{i} \\
& \mu_{i}=a_{i}+v_{i}
\end{aligned}
$$

com probabilidade $1-p$.

Observemos que no caso $k=1$, o modelo pode ser escrito usando notação matricial como

$$
\begin{aligned}
& \underline{y}=\underline{\mu}+\underline{\varepsilon}, \quad \underline{\varepsilon} \sim N\left(0, V_{1} I\right) \\
& \underline{\mu}=1_{n} \mu \\
& \mu \sim N(a, R)
\end{aligned}
$$

e sendo assim,$\underline{y} \mid \mu \sim N\left(1_{n} \mu, V_{1} I\right)$.

Consequentemente integrando fora $\mu$, temos

$$
\underline{y} \sim N\left(1_{n} a, 1_{n} R 1_{n}^{T}+V_{1} I\right)
$$


Se $k=0, \underline{y} \mid \underline{\mu} \sim N\left(\underline{\mu}, V_{1} I\right)$, e novamente integrando fora $\mu$, temos

$$
\underline{y} \sim N\left(\underline{a}, \operatorname{diag}\left(R_{1}, \cdots, R_{n}\right)+V_{1} I\right)
$$

onde $\underline{a}=\left(a_{1}, a_{2}, \cdots, a_{n}\right)$.

Portanto, na mistura teremos

$$
\underline{y} \sim(1-p) N\left(\underline{a}, \operatorname{diag}\left(R_{1}, \cdots, R_{n}\right)+V_{1} I\right)+p N\left(1_{n} a, 1_{n} R 1_{n}^{T}+V_{1} I\right) .
$$

E aqui, como no modelo (4.1), podemos escrever.

Para $k=1$ :

$$
\begin{aligned}
E\left(\mu_{i} \mid \underline{y}\right) & =E(\mu \mid \underline{y}) \\
& =\frac{R \bar{y}+V_{1} a}{V_{1}+R} \\
& =(1-q) \bar{y}+q a
\end{aligned}
$$

onde $q=V_{1} /\left(R+V_{1}\right)$, o que corresponde fazer $V_{2}=0$ em (4.1).

Para $k=0$ :

$$
\begin{aligned}
E\left(\mu_{i} \mid \underline{y}\right) & =\frac{R_{i} y_{i}+V_{1} a_{i}}{V_{1}+R_{i}} \\
& =\left(1-\alpha_{i}\right) y_{i}+\alpha_{i} a_{i}
\end{aligned}
$$

onde $\alpha_{i}=V_{1} /\left(V_{1}+R_{i}\right)$.

Portanto na mistura teremos

$$
E\left(\mu_{i} \mid \underline{y}\right)=(1-p)\left[\left(1-\alpha_{i}\right) y_{i}+\alpha_{i} a_{i}\right]+p[(1-q) \bar{y}+q a] .
$$

Observemos que se $R, R_{i} \rightarrow \infty$, ou seja, se tomarmos prioris difusas para $\mu, \mu_{i}$ no modelo (4.9),

$$
E\left(\mu_{i} \mid \underline{y}\right) \rightarrow(1-p) y_{i}+p \vec{y}
$$

que tem a forma de média ponderada.

Finalizaremos esta sessão fazendo um rápido comentário sobre a densidade a posteriori de $V_{1}$ e $p$ usando o modelo (4.9). 
Estimando e identificando na classe de modelos lineares hierárquicos normais

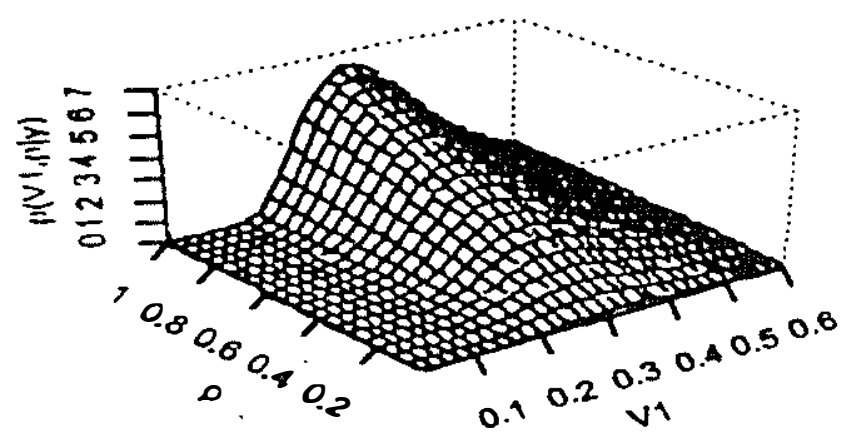

Figura la

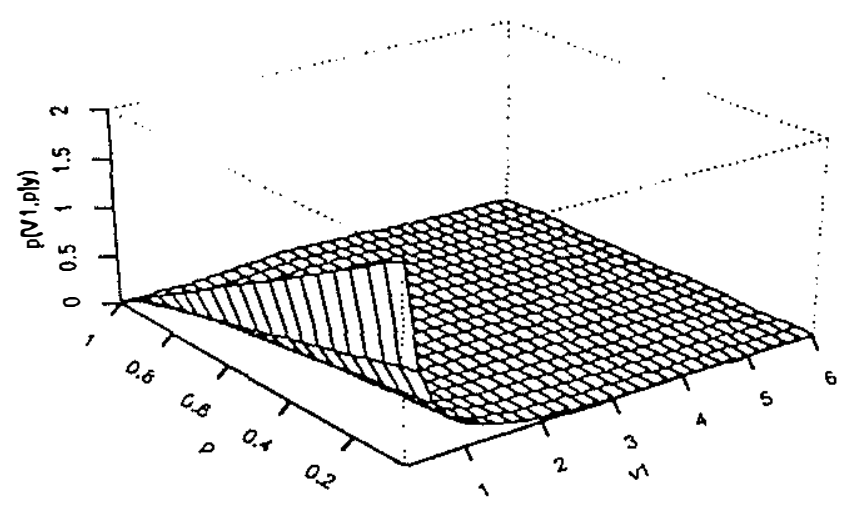

Figura $1 \mathrm{~b}$

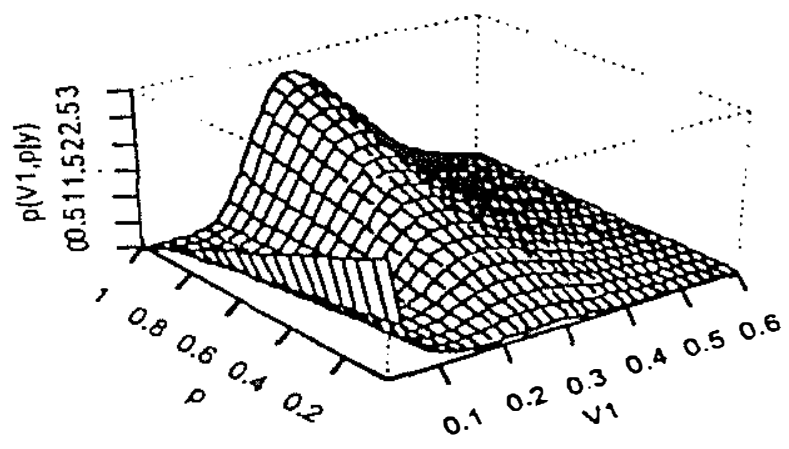

Figura 1c

FIGURA 1. Gráficos da Densidade a Posteriori para V1 e p - 
Os gráficos da figura 1 foram obtidos gerando para o modelo (4.9) um vetor de observações $(y)_{20 \times 1}$ usando:

(a) $p=0.7, R=R_{i}=4, a=10$ e $V_{1}=0.25$;

(b) $\quad p=0.7, R=R_{i}=4, a=10$ e $V_{1}=2.25$;

(c) $\quad p=0.7, R=4, R_{i}=1, a=10$ e $V_{1}=0,25$.

Da figura la observamos que a densidade é maior para valores grandes de $\mathrm{p}$, privilegiando assim o modelo em que todas as médias são iguais, ou seja, o modelo utilizado para gerar os dados. Na figura $1 \mathrm{~b}$ temos que a densidade é maior para valores pequenos de $p$, privilegiando assim o modelo em que as médias são diferentes. Este comportamento já era esperado pois da 1a equação de (4.9) temos que quanto maior for $V_{1}$ mais dispersos estarão os dados, ou seja, maior será o peso atribuído ao modelo com médias independentes. E finalmente da figura 1c, temos que na faixa contendo os valores plausíveis de $V_{1}$, isto é, contendo o valor usado para gerar os dados, o maior peso é atribuído para valores grandes de $p$ coincidindo com o comportamento do gráfico da figura 1a, privilegiando assim o modelo com médias iguais. Fora desta faixa, a densidade é maior para valores pequenos de $p$ tendo agora um comportamento semelhante ao da figura $1 \mathrm{~b}$, privilegiando agora o modelo com médias independentes. Em resumo, a figura 1c nos mostra que é possível fazer inferência sobre $V_{1}$ e $p$ usando o modelo (4.9). A figura 1 parece mostrar, apesar da correlação, ser possível separar as inferências de $V_{1}$ e $p$, diferentemente do modelo (4.1).

\section{Generalizações do Modelo $M_{1}$ Usando Mistura.}

$\mathrm{Na}$ seção anterior discutimos e apresentamos uma proposta para solucionar o problema de estimação de variância que ocorre no modelo (4.1). O modelo dinâmico $M_{1}$ é uma sucessão de modelos (4.1) e sendo assim, o problema de estimação de variâncias permanece. Nosso objetivo nesta seção é extender para o modelo 
Estimando e identificando na classe de modelos lineares hierárquicos normais

dinâmico $M_{1}$ o procedimento utilizado na seção anterior para sua versão estática.

Usando média ponderada, obtemos uma extensão do modelo $M_{1}$, dado por:

$$
\begin{aligned}
y_{t, i} & =\mu_{t, i}+\varepsilon_{t i, i}, \quad \varepsilon_{t i} \sim N\left(0, V_{1}\right) \\
\mu_{t, i} & =\left(1-p_{t,}\right) \mu_{t-1, i}+p_{t, \mu_{t}}+v_{t, i}, \quad v_{t, i} \sim N\left(0, V_{2}\right) \\
\mu_{t} & =\mu_{t-1}+\omega_{l,} \quad \omega_{l} \sim N(0, W) .
\end{aligned}
$$

Combinando as equações (5.1.1) e (5.1.2) podemos reescrever a equação das observações na forma

$$
y_{t, i}=\left(1-p_{t:}\right) \mu_{t-1, i,}+p_{t, \mu_{t}}+v_{t, i}+\varepsilon_{t, i} .
$$

Portanto este modelo, proposto por Gamerman \& Smith (1996), apresenta o mesmo problema de estimação das variâncias uma vez que a partir das observações só conseguimos estimar $V_{1}+V_{2}$. Além clisso os distúrbios $v_{t . i}^{\prime} s$ não tem um significado muito claro por serem combinações de evoluções individuais e evoluções dos níveis populacionais comuns. Esse modelo força reconciliação porque sua média está centrada numa combinação destes níveis. Uma proposta alternativa é obtida substituindo (5.1.2) por,

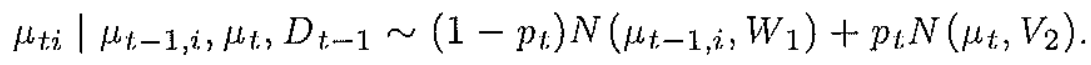

Observamos que:

1) Se $W_{1}=V_{2}$ e $\mu_{t-1, i}=\mu_{t}, \forall i$, o modelo (5.2) coincide com o modelo (5.1), mas, se $\mu_{t-1, i}$ estiver distante de $\mu_{t}$, o modelo não força reconciliação de níveis individuais e populacionais, ou seja, se estes níveis estão em conflito o modelo reflete esta discrepância.

2응 os distúrbios tem um significado estatístico uma vez que os níveis individuais e populacionais têm distribuições distintas. 
Usando variáveis indicadoras $k_{t}$, podemos reescrever o modelo (5.2) na forma

$$
\begin{aligned}
y_{t i,} & =\mu_{t i}+\varepsilon_{t, i} \\
\mu_{t i} & =\left(1-k_{t}\right)\left(\mu_{t-1, i}+\omega_{t i i}\right)+k_{t}\left(\mu_{t}+v_{t i}\right) \\
\mu_{t} & =\mu_{t,-1}+\omega_{t} \\
k_{t} & \sim \operatorname{Bern}\left(p_{t}\right) .
\end{aligned}
$$

Assim, o modelo oscila entre

$$
\begin{aligned}
y_{t i} & =\mu_{t i,}+\varepsilon_{t i} \\
\mu_{t, i} & =\mu_{t-1, i}+\omega_{t i}
\end{aligned}
$$

com probabilidade $1-p_{t}$ (e aqui temos o caso $\rho=1$ do modelo analisado em Klugman (1992)) e

$$
\begin{aligned}
y_{t, i} & =\mu_{t i}+\varepsilon_{t, i} \\
\mu_{t, i} & =\mu_{t}+v_{t, i} \\
\mu_{t} & =\mu_{t,-1}+\omega_{t}
\end{aligned}
$$

com probabilidade $p_{t}$ ( e aqui temos o modelo $M_{1}$ ).

Notemos que, se em (5.2), considerassemos $V_{2}=0$, teríamos o modelo

$$
\begin{aligned}
& y_{t, i}=\mu_{t, i}+\varepsilon_{t i}
\end{aligned}
$$

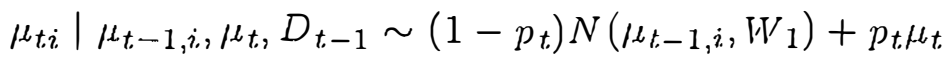

$$
\begin{aligned}
& \mu_{t}=\mu_{t-1}+\omega_{t}
\end{aligned}
$$

que é uma sucessão de modelos (4.9) analisados anteriormente e como foi visto não apresentam o problema de identificação do modelo (5.1). 
Estimando e identificando na classe de modelos lineares hierárquicos normais

Gamerman \& Smith (1996) consideraram possíveis formas de modelos dinâmicos para dados longitudinais combinando a informação estrutural em DHM via mistura. O modelo analisado por eles foi uma generalização do modelo de crescimento linear dada por

$$
\begin{aligned}
y_{t, i} & =\mu_{t i,}+\varepsilon_{t i} \\
\mu_{t, i} & =\left(1-p_{t}\right)\left(\mu_{t,-1, i}+\lambda_{t i,}\right)+p_{t} \mu_{t}+v_{1 t, i} \\
\lambda_{t, i} & =\left(1-p_{t .}\right) \lambda_{t-1, i}+p_{t:} \gamma_{t}+v_{2 t, i} \\
\mu_{t} & =\mu_{t-1}+\gamma_{t}+\omega_{1 t} \\
\gamma_{t} & =\gamma_{t-1}+\omega_{2 t}
\end{aligned}
$$

onde os $\mu_{t i i}^{\prime} s$ são os níveis individuais e os $\lambda_{t i}^{\prime} s$ são os parâmetros de crescimento. Esse modelo sofre os mesmos problemas que o modelo (5.1) e novamente essas dificuldades foram solucionadas, aproximando o modelo (5.4) via mistura de distribuições das médias e das taxas de crescimento tal como o modelo (5.2).

O modelo misturado é obtido substituindo-se (5.4.2) e (5.4.3) por:

$$
\begin{aligned}
& \mu_{t, i} \sim\left(1-p_{t}\right) N\left(\mu_{t-1, i}+\lambda_{t, i}, W_{11}\right)+p_{t, N}\left(\mu_{t}, V_{21}\right) \\
& \lambda_{t, i} \sim\left(1-p_{t .}\right) N\left(\lambda_{t-1, i}, W_{12}\right)+p_{t,} N\left(\gamma_{t,}, V_{22}\right) .
\end{aligned}
$$

Essa mistura não força reconciliação de níveis individuais e populacionais, ou seja, se estes níveis estão em conflito, o modelo reflete esta discrepância, caso contrário o modelo se comporta tal como em (5.4).

\section{Extensões dos Modelos Estudados.}

Seguindo o que foi feito para o modelo polinomial de $1^{\underline{a}}$ ordem obtemos os modelos alternativos apresentados abaixo, que são extensões de modelos polinomiais de ordem $k$ e modelos de regressão. 


\section{Eliane A. Camargo \& Dani Gamerman}

Para o modelo de crescimento linear dado por

$$
\begin{aligned}
y_{t, i} & =\mu_{t, i}+\varepsilon_{t, i} \\
\mu_{t, i} & =\mu_{t,}+v_{t i} \\
\mu_{t} & =\mu_{t-1}+\gamma_{t}+w_{t, 1} \\
\gamma_{t} & =\gamma_{t-1}+w_{t, 2}
\end{aligned}
$$

obtemos misturando somente as médias

$$
\begin{aligned}
y_{t, i} & =\mu_{t, i}+\varepsilon_{t i} \\
\mu_{t, i} & \sim(1-p) N\left(\mu_{t,-1, i}+\lambda_{t, i}, W_{11}\right)+p \mu_{t} \\
\lambda_{t, i} & =\lambda_{t-1, i}+w_{t, i 2}, \quad w_{t, i 2} \sim N\left(0, W_{12}\right) \\
\mu_{t} & =\mu_{t-1}+\gamma_{t}+w_{t, 1}, \quad w_{t, 1} \sim N\left(0, W_{1}\right) \\
\gamma_{t} & =\gamma_{t-1}+w_{t, 2}, \quad w_{t, 2} \sim N\left(0, W_{2}\right)
\end{aligned}
$$

onde os distúrbios $w_{t, j}^{\prime} s$ são normalmente distribuídos e mutuamente não correlacionados. Se por outro lado misturarmos somente as taxas de crescimento, obtemos

$$
\begin{aligned}
y_{t, i} & =\mu_{t, i}+\varepsilon_{t, i} \\
\mu_{t, i} & =\mu_{t-1, i}+\lambda_{t, i}+w_{t, i 1} \\
\lambda_{t, i} & \sim\left(1-p_{t}\right) N\left(\lambda_{t-1, i}, W_{12}\right)+p_{t,} \gamma_{t} . \\
\gamma_{t} & =\gamma_{t-1}+\omega_{t,} .
\end{aligned}
$$

As mesmas idéias podem ser extendidas a modelos de regressão com estrutura hierárquica. Para o modelo de regressão estático dado por:

$$
\begin{aligned}
y_{i} & =\underline{x}_{i}^{T} \underline{\beta}_{i}+e_{i}, \quad e_{i} \sim N\left(0, V_{1}\right) \\
\underline{\beta}_{i} & =\underline{\beta}+\underline{v}_{i}, \quad v_{i} \sim N\left(0, V_{2}\right) \\
\beta & \sim N(a, R)
\end{aligned}
$$


temos que $y_{i}$ é uma variável observável, cujo valor é afetado por variáveis $x_{i}^{\prime} s$, chamadas de variáveis explicativas. A presença destas variáveis explicativas torna o modelo identificável pois o modelo pode ser reescrito na forma

$$
y_{i}=\underline{x}_{i}^{T} \underline{\beta}+\xi_{i}, \quad \operatorname{Var}\left(\xi_{i}\right)=x_{i}^{T} V_{2} x_{i}+V_{1} .
$$

O modelo alternativo usando mistura de distribuições é dado por

$$
\begin{aligned}
y_{i} & =\underline{x}_{i}^{T} \underline{\beta}_{i}+e_{i} \\
\underline{\beta}_{i} & \sim(1-p) N\left(\underline{a}_{i}, R_{i}\right)+p \underline{\beta} \\
\underline{\beta} & \sim N(a, R) .
\end{aligned}
$$

Neste modelo substituímos a variância $V_{2}$ pelo parâmetro $p$.

A versão dinâmica para modelos de regressão, usanclo mistura, nos dá:

$$
\begin{aligned}
y_{t i} & =\underline{x}_{t i}^{T} \beta_{t, i}+e_{t i} \\
\beta_{t i} & \sim(1-p) N\left(G_{i} \beta_{t-1, i}, W_{11}\right)+p \beta_{t} \\
\underline{\beta}_{t} & =G \beta_{t-1}+\omega_{l, .}
\end{aligned}
$$

Essas idéias foram extendidas também para modelos polinomiais de ordem $k$ por Camargo (1995).

Vale ressaltar que como o modelo linear de $1 \underline{a}$ ordem ilustra conceitos básicos e características importantes da classe de Modelo Linear Normal Dinâmico, toda a análise feita para este modelo pode ser extendida para os modelos alternativos sugeridos acima.

\section{Conclusão.}

Como foi dito na introdução o objetivo deste trabalho foi detectar e analisar problemas de identificação, estimação e previsão que ocorrem particularmente no modelo $M_{1}$, que é um modelo que 
apresenta variações em cada tempo para acomodar as diferenças individuais das séries e evoluções no tempo representados por um passeio aleatório.

Utilizando mistura de normais, modificamos o modelo $M_{1}$ de modo a torná-lo identificável possibilitando assim estimativas mais eficientes. Levando em conta que este modelo pode ser visto como um elemento gerador da classe de Modelos Lineares Normais Dinâmicos (DLMs), esta modificação sugere um método para obter extensões de modelos polinomiais de ordem $\mathrm{k}$ e modelos de regressão e, consequentemente todo ganho obtido para o modelo $M_{1}$ poderá ser repassado para estas extensões. Gostaríamos de ressaltar que a extensão do modelo $M_{1}$ usando mistura não é possível apenas para observações normais. Atualmente trabalhamos com uma modificação deste modelo para observações com distribuições binomiais que mistura informações individuais e populacionais utilizando distribuições betas.

Submetido em Setembro de 1995. Revisado em Outubro de 1996.

\section{Referências}

Camargo, E. A. 1995. "Modelos Multivariados de Tendência. Exame de Qualificação". COPPE/UFRJ, Programa de Engenharia de Produção.

Engle, R. F. \& Granger, C. W. J. 1987. "Co-integration and error correction: representation, estimation and testing". Econometrica. 55: 251-276.

Gamerman, D. 1992. "Multivariate trend models". Trabalho apresentado no U.S. - Latinamerican Workshop on Bayesian Statistics and Econometrics, Caracas. Também Relatório Técnico no. 61 do Laboratório de Estatística do IM/UFRJ.

Gamerman, D. \& Migon, H. S. 1993a. "Dynamic hierarchical models". Journal of the Royal Statistical Soc.. Serie B, 55: 629-642. 
Estimando e identificando na classe de modelos lineares hierárquicos normais

Garmerman, D. \& Migon, H. S. 1993b. "An application of dynamic hierarchical models to forecasting exports". Trabalho apresentado no 1st. Riverboat Conference on Bayesian Econometrics and Statistics, Basel-Amsterdam.

Gamerman, D. \& Smith, A. F. M. 1996. "Bayesian analysis of longitudinal data studies". In Bernardo, J.M. et al. eds. Em Bayesian Statistics. 5: 587-597, Oxford University Press.

Godolphin, E. J. \& Harrison, P. J. 1975. "Equivalence theorems for polynomial projecting predictors". Journal of the Royal Statistical Society. Serie B, 37: 205-215.

Harrison, P. J. 1965. "Short-term sales forecasting". Applied Statistics. 15: 102-139.

Harvey, A. C. 1989. Forecasting, Structural Time Series Models and the Kalman Filter. Cambridge University Press.

Issler, J. V. 1992. Resenha do livro: "Long-Run Economic Relationships: Readings in Cointegration". Revista de Econometria. 12: 241-248.

Klugman, S. A. 1992. Bayesian Statistics in Actuarial With Emphasis on Credibility. Kluwer Academic Publishers.

Marshall, P. 1991. "Estimating time-dependent means in dynamic models for cross-section of time series". Empirical Economics. 17: 25-33.

Nelson, C. R. \& Plosser, C. I. 1982. "Trends and random walks in macroeconomic time series". Journal of Monetary Economics. 10: $139-162$.

West, M. \& Harrison, P. J. 1989. Bayesian Forecasting and Dynamic Models. New York: Springer-Verlag.

Zellner, A. 1971. An Introduction to Bayesian Inference in Econometrics. John Willey \& Sons,Inc. 